\title{
Reward Sensitivity of ACC as an Intermediate Phenotype between DRD4-521T and Substance Misuse
}

\author{
Travis E. Baker ${ }^{1,2}$, Tim Stockwell ${ }^{1}$, Gordon Barnes ${ }^{1}$, Roderick Haesevoets ${ }^{1}$, \\ and Clay B. Holroyd ${ }^{1}$
}

\begin{abstract}
The development and expression of the midbrain dopamine system is determined in part by genetic factors that vary across individuals such that dopamine-related genes are partly responsible for addiction vulnerability. However, a complete account of how dopamine-related genes predispose individuals to drug addiction remains to be developed. Adopting an intermediate phenotype approach, we investigated whether reward-related electrophysiological activity of ACC - a cortical region said to utilize dopamine reward signals to learn the value of extended, context-specific sequences of goal-directed behaviors-mediates the influence of multiple dopamine-related functional polymorphisms over substance use. We used structural equation modeling to examine whether two related electrophysiological phenomena associated with the control and reinforcement learn-
\end{abstract}

\section{INTRODUCTION}

Despite the fact that substance use disorders are partly heritable (Johnson et al., 2008; Uhl et al., 2008), compelling evidence linking individual genes with the disorders remains elusive. One strategy for addressing complex gene-disease relationships depends on the concept of intermediate phenotypes (IPs): biological and psychological factors that are relatively proximal to genetic influence and confer vulnerability to (rather than determine) psychopathology (Meyer-Lindenberg \& Weinberger, 2006). It has been suggested that IPs should be defined in relation to the cardinal features of a disorder such as a subclinical variant of a form of major psychopathology ${ }^{1}$ (Lenzenweger, 2013; Loth, Carvalho, \& Schumann, 2011; Meyer-Lindenberg \& Weinberger, 2006). In the case of substance misuse, the consumption of drugs and alcohol is considered problematic when it imposes a significant cost on the individual, is difficult to interrupt, is likely to recur after interruption, and continues despite long-term adverse consequences (Lima et al., 2015) characteristics that are strongly suggestive of impaired cognitive control ${ }^{2}$ (Hyman, Malenka, \& Nestler, 2006). This behavioral profile therefore suggests the identification of

${ }^{1}$ University of Victoria, ${ }^{2}$ University of Montreal

(C) 2016 Massachusetts Institute of Technology ing functions of ACC - theta power and the reward positivitymediated the relationship between the degree of substance misuse and genetic polymorphisms that regulate dopamine processing in frontal cortex. Substance use data were collected from 812 undergraduate students. One hundred ninety-six returned on a subsequent day to participate in an electrophysiological experiment and to provide saliva samples for DNA analysis. We found that these electrophysiological signals mediated a relationship between the DRD4-521T dopamine receptor genotype and substance misuse. Our results provide a theoretical framework that bridges the gap between genes and behavior in drug addiction and illustrate how future interventions might be individually tailored for specific genetic and neurocognitive profiles.

IPs that relate to the expression of flexible and adaptive goal-directed behaviors in this population.

In this study, we utilize the cognitive control function of ACC as an IP that elucidates the genetic contribution to substance misuse. Although the specific role of ACC in control is highly controversial, a recent theory holds that ACC motivates the execution of extended, goal-directed sequences of behavior according to principles of hierarchical reinforcement learning (Holroyd \& McClure, 2015; Holroyd \& Yeung, 2012), a process that is said to be facilitated by the impact of dopamine reward prediction error signals on ACC (RPE-ACC theory; Holroyd \& Coles, 2002). RPEs constitute the learning term in powerful reinforcement learning algorithms that indicate when events are "better" or "worse" than expected (Sutton \& Barto, 1998). Current thinking holds that positive and negative RPEs are encoded as phasic increases and decreases in the firing rate of midbrain dopamine neurons, respectively (Schultz, 2010, 2011, 2013). The RPE-ACC theory extends this concept by proposing that ACC utilizes negative and positive RPEs to learn the value of extended, context-specific sequences of behavior directed toward particular goals (Holroyd \& Yeung, 2012).

ACC is also strongly implicated in addiction (Peoples, 2002), but its contribution to this disorder is still relatively unknown. Extensive empirical and theoretical work 
has shown that addictive drugs potentiate dopaminergic RPE signals (Schultz, 2011; Redish, Jensen, \& Johnson, 2008; Redish, 2004) by raising extracellular dopamine levels either directly or indirectly (Di Chiara \& Bassareo, 2007; Rice \& Cragg, 2004; Di Chiara \& Imperato, 1988). Although much attention has focused on the impact of aberrant dopaminergic signaling on the function of the striatum (Volkow, Wang, Fowler, \& Tomasi, 2012; Volkow, Wang, Fowler, Tomasi, \& Telang, 2011), a region commonly thought to learn and implement relatively simple stimulus-response associations (Yin \& Knowlton, 2006), this altered processing likely extends to ACC, an important neural target of the dopamine system (Gaspar, Berger, Febvret, Vigny, \& Henry, 1989). In the context of the RPE-ACC theoretical framework, we have argued that the distortion of RPE signals by addictive drugs would upset the normal function of ACC, biasing the action selection mechanism to favor extended behaviors that ultimately converge on drug use (Baker, 2012; Baker, Stockwell, Barnes, \& Holroyd, 2011).

Several genetic studies have also implicated dopamine and ACC in cognitive control and addiction. In particular, functional polymorphisms of the DRD4 gene, which plays a fundamental role in the overall inhibitory modulation of frontal cortex neuronal activity (Onn, Wang, Lin, \& Grace, 2006; Rubinstein et al., 2001), and the catecholO-methyltransferase (COMT) gene, which plays a crucial role in the metabolism of dopamine in frontal cortex (Chen et al., 2004), modulate cognitive control functions related to ACC (Marco-Pallares et al., 2009; Kramer et al., 2007). For example, DRD4 functional polymorphismsnamely, the promoter $-521 \mathrm{C} / \mathrm{T}$ (rs1800955) and the Indel $-1217 \mathrm{G}$ ins/del (-/G) (rs12720364) single-nucleotide genetic polymorphisms (SNPs) of the DRD4 gene (Oak, Oldenhof, \& Van Tol, 2000) — are associated with ACC function (Fan, Fossella, Sommer, Wu, \& Posner, 2003; Oak et al., 2000) and appear to modulate electrophysiological markers of feedback processing (Agam et al., 2014; Manoach \& Agam, 2013; Marco-Pallares et al., 2010; MarcoPallares et al., 2009). Furthermore, the val158met (rs4680) SNP of the COMT gene, which accounts for a fourfold decrease in catabolism of dopamine and other catecholamines in frontal cortex (Chen et al., 2004), has been linked to cognitive flexibility and working memory functions related to frontal cortex (Dickinson \& Elvevag, 2009). Importantly, functional polymorphisms of the DRD 4 and COMT gene have also been implicated in addiction, including alcohol craving, and alcohol, nicotine, opioid, and cannabis misuse (McGeary, 2009; Ray et al., 2009; Baransel Isir et al., 2008; Oosterhuis et al., 2008; Mackillop, Menges, McGeary, \& Lisman, 2007; Beuten, Payne, Ma, \& Li, 2006; Hutchison, McGeary, Smolen, Bryan, \& Swift, 2002; Oak et al., 2000).

Neuroimaging-based measures have emerged as particularly promising candidates for assessing IPs because they reveal neurocognitive processes that can be altered in clinical disorders (Rasetti \& Weinberger, 2011). Here, we utilize two related electrophysiological signals associated with ACC function-theta power and the reward positivity (RP) - to serve as indices of our proposed ACCrelated IP. Both of these phenomena have been extensively studied in parallel, decade-long literatures. With regard to the former signal, EEG oscillations in the theta frequency range $(4-8 \mathrm{~Hz})$ recorded over frontal midline areas of the scalp have, since the 1970 s, been associated with cognitive process related to effort, attention, and motivation (for reviews, see Hsieh \& Ranganath, 2014; Mitchell, McNaughton, Flanagan, \& Kirk, 2008). Furthermore, unexpected, task-relevant events elicit a brief burst of power in the theta frequency range about 200-300 msec after the event that appears to index the deployment of control (Cavanagh \& Frank, 2014; Cavanagh, ZambranoVazquez, \& Allen, 2012). With respect to the latter signal, the RP is observed as a differential response in the ERP to positive and negative feedback stimuli, occurring over frontal-central areas of the scalp about 250-300 msec after feedback (Miltner, Braun, \& Coles, 1997). The RPE-ACC theory holds that the RP is produced by the impact of phasic increases and decreases in dopamine activity coding for positive and negative RPEs, respectively, on ACC (Holroyd, Pakzad-Vaezi, \& Krigolson, 2008; Holroyd \& Coles, 2002). Substantial evidence over the past decade has confirmed that the RP reflects an RPE signal (for reviews, see Sambrook \& Goslin, 2015; Walsh \& Anderson, 2012).

Furthermore, converging evidence across multiple methodologies indicates that ACC is the source of both frontal midline theta oscillations (Holroyd, 2015) and the RP (Holroyd \& Yeung, 2012). The fact that feedbackinduced theta power and the RP occur at about the same time (200-300 msec after feedback) and share the same scalp location (over the frontal midline) suggests a functional relationship between these two phenomena. In particular, recent examinations of the RP and theta power have provided a nuanced account about their relationship (Hajihosseini \& Holroyd, 2013). On this account, unexpected, task-relevant events elicit an ACC-dependent control process that manifests in the frequency domain as theta oscillations over frontal-central areas of the scalp (Cavanagh \& Frank, 2014). In the time domain, the "evoked" portion of this theta activity that is consistent in phase across trials gives rise to an ERP component called the N200 (Hajihosseini \& Holroyd, 2013; see also Yeung, Bogacz, Holroyd, \& Cohen, 2004). This account holds that both theta and the N200 constitute a baseline response to unexpected events and, furthermore, that N200 amplitude is regulated up and down by dopamine RPE signals conveyed to ACC. Thus, positive dopamine RPE signals conveyed to ACC about 300 msec after unexpected positive feedback (Zaghloul et al., 2009) suppresses the production of the N200 (Holroyd, Krigolson, \& Lee, 2011; Holroyd et al., 2008; see Proudfit, 2015, for a review), whereas negative dopamine RPE signals enhance N200 amplitude (Warren \& Holroyd, 2012). For this reason, 
the RP is typically operationalized as the size of the difference wave calculated between good (positive RPE) and bad (negative RPE) outcome ERPs (Sambrook \& Goslin, 2015; Holroyd \& Krigolson, 2007; Miltner et al., 1997).

In view of the close relationship between these phenomena, we included both theta and the RP as a combined index of our proposed ACC-related IP. Note that the amplitude of the RP is heritable and has excellent test-retest reliability, indicating that the RP provides a stable, trait-like neural measure (Weinberg \& Hajcak, 2011; Olvet \& Hajcak, 2008) or a susceptibility-related phenotype (Proudfit, 2015) of the underlying reward process. Furthermore, we have previously reported that the RP was attenuated in undergraduate students meeting criteria for substance dependence, which is suggestive of disrupted dopamine RPE signaling in this population (Baker et al., 2011). In fact, numerous studies have shown the RP to be abnormal in several psychiatric disorders (Proudfit, 2015), consistent with the idea that ACC may implement a crucial neurocognitive function that cuts across traditional diagnostic categories (Holroyd \& Umemoto, 2015; see also Cuthbert \& Insel, 2013; Insel et al., 2010).

We propose that ACC's putative function-selecting and motivating the execution of goal-directed extended sequences of actions based on reinforcement learning principles_can be utilized as an IP for the abnormal cognitive control processes underlying individual differences in substance misuse. In particular, we propose that SNPs that regulate the expression of dopamine processes in frontal cortex (i.e., DRD4, COMT) may contribute to the degree of substance misuse observed in undergraduate students by modulating ACC activity as revealed in individual differences in RP amplitude and theta power. To test this idea, we measured the RP and theta power from first- and second-year undergraduate students while they navigated a virtual T-maze by trialand-error to find rewards as in our previous study (Baker et al., 2011). All participants were screened for problematic substance use and provided saliva samples for DNA analysis. Importantly, we applied structural equation modeling (SEM) to examine whether theta power and RP amplitude would show an intermediate relationship between several dopamine-related genetic polymorphisms (i.e., the promoter - 521 [rs1800955] and indel $-1217 \mathrm{G}$ [rs12720364] SNP of the DRD4 gene and val158met polymorphism [rs4680] of the COMT gene) and the degree of substance misuse in undergraduate students. The IP approach constitutes a natural application of SEM, which provides a means for modeling such complex interrelationships.

\section{METHODS}

\section{Participants}

In keeping with a previous experiment (Baker et al., 2011), we collected questionnaire data from 812 undergraduate students recruited from the University of Victoria,
Table 1. Genotype Characteristics of the Research Sample Population

\begin{tabular}{lccccc}
\hline Genetic & Characteristics $(n=195)$ & & \\
\hline Gene & SNP & SNP rs\# & \multicolumn{2}{c}{ Allele/Phenotype/Sample } \\
\hline DRD4 & C-521T & 1800955 & CC $\uparrow$ & CT $\uparrow \downarrow$ & TT $\downarrow \downarrow$ \\
& & & $n=43$ & $n=107$ & $n=45$ \\
& & & & \\
& Indel & 12720364 & GG & $-\mathrm{G}$ & $-/-^{\mathrm{a}}$ \\
& & & $n=75$ & $n=89$ & $n=29$ \\
COMT & Val158Met & 4680 & $\mathrm{MM} \downarrow \downarrow$ & $\mathrm{MV} \uparrow \downarrow$ & $\mathrm{VV} \uparrow$ \\
& & & $n=54$ & $n=101$ & $n=40$ \\
\hline
\end{tabular}

$\uparrow \downarrow$ denotes an increase or decrease in dopaminergic phenotype.

${ }^{\mathrm{a}}$ Phenotype currently unknown.

each of whom received course credit in a psychology course for their participation. Participants were allowed $60 \mathrm{~min}$ to complete the questionnaire in a computer laboratory at the University of Victoria, and up to 20 participants completed the questionnaire at a time. They were provided with both written and verbal instructions that explained the procedure and were instructed that they should answer each of the questions truthfully and to the best of their knowledge. The computer-based questionnaire was composed of several separate inventories that assess problematic drug use, the degree of addiction vulnerability, and personality risk factors for substance dependence. At the end of the questionnaire, participants were asked whether they would like to return on a subsequent day to participate in an electrophysiological and behavioral experiment and to provide saliva samples for DNA analysis (Table 1). Of these 812 participants, 196 agreed to participate in the subsequent experiment; this proportion of returning participants (24\%) was comparable with that of our previous study (17\%; Baker et al., 2011). The data of one participant were excluded because of a contaminated DNA sample. All remaining participants had normal or corrected-to-normal vision, and all participants gave informed consent. The study was approved by the local research ethics committee and was conducted in accordance with the ethical standards prescribed in the 1964 Declaration of Helsinki.

For the purpose of this study, we defined the level of substance misuse as frequency of substance use behaviors that impose a significant cost on the individual, are difficult to interrupt, and are likely to recur after interruption. Substance misuse by this definition was measured as a continuous variable using the Global Continuum of Substance Risk (GCR) score of the World Health Organization Alcohol, Smoking and Substance Involvement Screening Test (ASSIST; Humeniuk et al., 2008). The ASSIST is a validated screening test using DSM-specific criteria for identifying the degree of problematic substance use 
(i.e., tobacco, alcohol, cannabis, cocaine, amphetamine-type stimulants, sedatives, hallucinogens, inhalants, opioids, and "other drugs"), especially in individuals who consume a variety of different substances with varying degrees of use, for example, as occurs with polydrug use. Usages of individual substances were each scored according to the ASSIST guidelines (Humeniuk et al., 2008), and scores were combined to derive the GCR score. To be specific, the GCR score was based on the sum of response weights to Questions 1-8 (1. Have you ever used Substance A? 2. Used in the past 3 months? 3. During the past 3 months, how often have you had a strong desire or urge to use this substance? 4 . During the past 3 months, how often has your use of this substance led to health, social, legal, or financial problems? 5 . During the past 3 months, how often have you failed to do what was normally expected of you because of your use of this substance? 6. Has a friend or relative or anyone else EVER expressed concern about your use of this substance? 7. Have you ever tried and failed to control, cut down, or stop using this substance? 8. Have you ever used any drug by injection?) across the following drug classes: tobacco, alcohol, cannabis, cocaine, amphetamine-type stimulants, sedatives, hallucinogens, inhalants, opioids and "other drugs."

Importantly, the ASSIST allows for generalization of results across substance-dependent (SD) populations and is less dependent on the distribution of any given sample (Humeniuk et al., 2008). As reported in a validation study by Newcombe, Humeniuk, and Ali (2005), the significant correlation between the GCR score and the score derived from the Severity of Dependence Scale suggests that the GCR score is a valid measure of severity of dependence for the individual concerned. Following the screening procedure utilized in our previous study of substance dependence (Baker et al., 2011), personality risk factors for substance dependence were also assessed, but these factors were not the focus of this investigation and are discussed elsewhere (Baker, Stockwell, \& Holroyd, 2013). All information obtained was kept strictly confidential and stored in a locked filing cabinet.

\section{Genotyping}

Purified DNA was obtained from participants by collecting and processing saliva samples using Oragene OG500 DNA Collection Kits (DNA Genotek, Ottawa, Canada) as per the manufacturer's instructions. Each participant's sample was processed at the Center for Biomedical Research DNA Sequencing Facility, University of Victoria. Several separate regions of genomic DNA, each containing a polymorphism of interest, were amplified by polymerase chain reaction and purified. Genotyping of the DRD4 C-521T and DRD4 - 127 Indel SNPs was accomplished by digestion with a restriction endonuclease appropriate to each polymorphism and subsequent separation by agarose gel electrophoresis. Genotyping of the COMT Val156Met polymorphisms was accomplished by tetra-primer amplification refractory mutation systempolymerase chain reaction and subsequent separation by agarose gel electrophoresis. Each genotyping method for each SNP target was verified by performing DNA sequence analysis (LI-COR 4200 Genetic Analyzer, Lincoln, NE) on a representative subset of samples. Genotyping of SNPs (DRD2 Taqa1, PPP1R1B rs879606, PPP1R1B rs907094, DRD2 C957T, and DRD2 rs12364283) related to behavioral performance on a decision-making task (Frank, Seeberger, O'Reilly, 2004) was also performed, the results of which are reported elsewhere (Baker et al., 2013; Baker, 2012).

\section{Electrophysiological Task: Virtual T-Maze}

The virtual T-maze is a reward-based choice task that elicits robust RPs (Baker et al., 2011; Baker \& Holroyd, 2009). At the start of each block of trials, participants were shown three different aerial views of the maze, each for $3 \mathrm{sec}$, to familiarize themselves with its virtual dimensions (Figure 1, top row). On each trial, participants were presented with an image of the base arm of a T-maze showing the length of the arm and two alleys projecting to the left and to the right from its far end (Figure 1, bottom). Participants were instructed to choose one of the two arms by pressing either a left or right button. Then, they were shown a view of the alley that they selected, followed by an image of either an apple or an orange appearing against the far wall of that alley. Participants were told that presentation of one type of fruit indicated that the alley they selected contained 5 cents (Reward feedback) and that the presentation of the other fruit indicated that the alley they selected was empty (Noreward feedback); the mappings between feedback stimuli and reward types were counterbalanced across participants. Participants were also informed that, at the

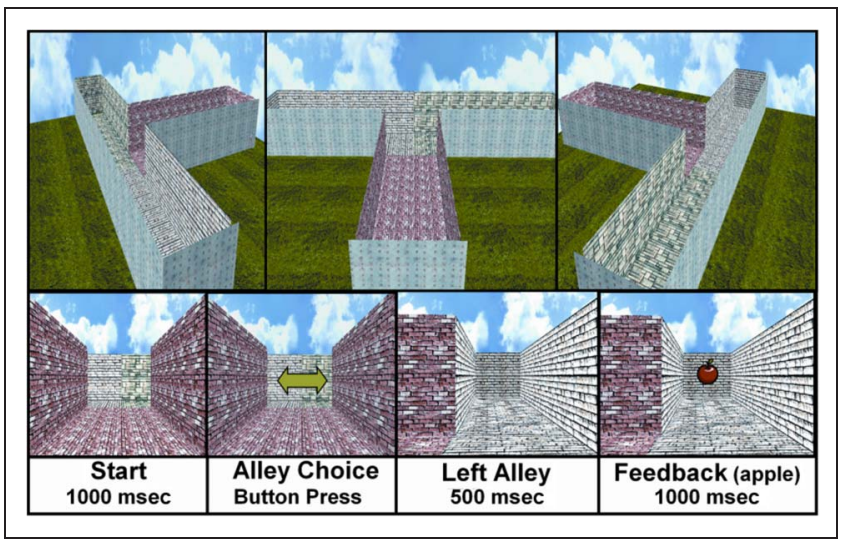

Figure 1. The virtual T-maze task. (Top) Three views of T-maze from above. (Bottom) Sequence of events constituting an example trial of the T-maze task; stimulus durations are indicated at the bottom of each panel. The double arrow remained visible until the button press. Please note that the size of the arrow was magnified in this figure for the purpose of exposition. 
end of the experiment, they would be rewarded all the money they found and that they were to respond in a way that maximized the total amount of money earned. Unbeknownst to them, on each trial, the type of feedback stimulus was selected at random (50\% probability for each feedback type, which is a standard probability used to elicit a robust RP). The experiment consisted of four blocks of 100 trials each separated by rest periods. Upon completing the T-maze, participants engaged in a decision-making task (Frank et al., 2004), the results of which are reported elsewhere (Baker et al., 2013). At the end of the experiment, participants were informed about the probabilities and were given a $\$ 10$ performance bonus.

\section{Data Acquisition}

EEG was recorded using a montage of 36 electrodes placed according to the extended international 10-20 system (Jasper, 1958). Signals were acquired using Ag$\mathrm{AgCl}$ ring electrodes mounted in a nylon electrode cap with a conductive gel (Falk Minow Services, Herrsching, Germany). Signals were amplified by low-noise electrode differential amplifiers with a frequency response of DC $0.017-67.5 \mathrm{~Hz}$ (90-dB octave roll-off) and digitized at a rate of 250 samples per second. Digitized signals were recorded to disk using Brain Vision Recorder software (Brain Products GmbH, Munich, Germany). Electrode impedances were maintained below $10 \mathrm{k} \Omega$. Two electrodes were also placed on the left and right mastoids. The EEG was recorded using the average reference. For the purpose of artifact correction, the horizontal EOG was recorded from the external canthi of both eyes, and vertical EOG was recorded from the suborbit of the right eye and electrode channel Fp2.

\section{Data Analysis}

Postprocessing and data visualization were performed using Brain Vision Analyzer software (Brain Products $\mathrm{GmbH})$. The digitized signals were filtered using a fourth-order digital Butterworth filter with a bandpass of 10-20 Hz. An 800-msec epoch of data extending from $200 \mathrm{msec}$ before to $600 \mathrm{msec}$ after the onset of each feedback stimulus was extracted from the continuous data file for analysis. Ocular artifacts were corrected using the eye movement correction algorithm described by Gratton, Coles, and Donchin (1983). The EEG data were rereferenced to linked mastoids electrodes. The data were baseline-corrected by subtracting from each sample the mean voltage associated with that electrode during the 200-msec interval preceding stimulus onset. Muscular and other artifacts were removed using a $\pm 150-\mu \mathrm{V}$ level threshold and $\mathrm{a} \pm 35-\mu \mathrm{V}$ step threshold as rejection criteria. ERPs were then created for each electrode and participant by averaging the single-trial EEG according to feedback type (Reward, No-reward).

\section{Reward Positivity}

$\mathrm{RP}$ amplitude is typically assessed as the size of the difference in the ERPs elicited to positive and negative feedback stimuli of equal expectancy (Sambrook \& Goslin, 2015; Holroyd \& Krigolson, 2007). Thus, to isolate the RP from other overlapping ERP components, the RP was evaluated for each electrode channel and participant as a difference wave by subtracting the Reward feedback ERPs from the corresponding No-reward feedback ERPs (Sambrook \& Goslin, 2015; Holroyd \& Krigolson, 2007; Miltner et al., 1997). The size of the RP was then determined by identifying the peak amplitude of the difference between the Reward and No-reward ERPs within a 200- to 400-msec window after feedback onset. For statistical analysis, the RP was measured at channel $\mathrm{FCz}$, where it reaches maximum amplitude (Holroyd \& Krigolson, 2007; Miltner et al., 1997).

\section{Theta Power}

Theta power was obtained by computing a single-trial wavelet-based time-frequency analysis using customwritten MATLAB routines that implement the method described by Lachaux, Rodriguez, Martinerie, and Varela (1999). The continuous EEG data were segmented in 5000-msec epochs (2500 msec preceding to $2500 \mathrm{msec}$ after feedback presentation) and filtered using a fourthorder digital Butterworth filter with a bandpass from 0.1 to $40 \mathrm{~Hz}$. Single-trial EEG data were convoluted with a complex seven-cycle Morlet wavelet in the frequencies of interest (from 1 to $40 \mathrm{~Hz}$; linear increase in steps of $2 \mathrm{~Hz}$ ) averaged for each participant before performing a grand average. The relative change in the power for each condition was determined by averaging the baseline activity (100-msec prestimulus: -300 to $-200 \mathrm{msec}$ ) across time for each frequency and then subtracting the average from each data point after stimulus presentation for the corresponding frequency (for a complete description of the analysis procedure, see Hajihosseini \& Holroyd, 2013). Each epoch was then separated into epochs extending from -500 to $+1000 \mathrm{msec}$ after stimulus onset. For each participant, we calculated the mean power for theta $(4-8 \mathrm{~Hz})$ within a temporal window extending across this interval, and the peak power and latency were obtained by detecting power maximum within a 600-msec window after the onset of the feedback stimulus. This analysis was restricted to channel FCz where frontal-midline theta is maximal (Cavanagh \& Shackman, 2015; Hajihosseini \& Holroyd, 2013).

\section{Statistical Analysis}

Data analysis was performed using SPSS 17.0.1 for Windows (SPSS, Inc., Chicago, IL), and the SEM was tested and modified using the computer program AMOS 18.0.1 (Arbuckle, 1995-2009). Descriptive statistics were obtained to 
characterize the sample (Table 1). SEM is a confirmatory modeling technique for theory development and testing. Confirmatory modeling entails operationalizing a hypothesis in a causal model to test specific interrelationships between elements of the hypothesis. Here, we examined whether our theoretically constrained IP model (in which the relationships between genes and IP measures were "fixed" based on observations from previous studies) could adequately account for the contribution of several dopamine-related genes to substance dependence. In particular, we examined whether theta power and RP amplitude could account for the contribution of DRD4 C-521T, DRD4 -127 Indel SNPs, and COMT Val156Met polymorphisms to substance misuse as defined by the GCR score (the degree of problematic substance use). To be specific, GCR scores were included in this model as the outcome measure, with the RP (evaluated as the size of the difference wave taken between Reward and No-reward ERPs) and total theta power $(\theta)$ elicited by feedback during the virtual T-maze task as first-order predictor variables (Figure 2). Furthermore, given the hypothesis that phasic dopamine signals modulate the amplitude of ACC-generated theta oscillations to produce the RP (Hajihosseini \& Holroyd, 2013), we allowed theta power to predict the amplitude of the RP in the proposed model (Figure 2). The paths between the functional polymorphisms and the IP were based on their previous associations with frontal cortical function.

The ACC-IP model was tested and modified (if needed) using the computer program AMOS 18.0.1 (Arbuckle, 1995-2009), which estimated the error terms of the directly observable variables that were assumed to be uncorrelated with each other. We examined the indirect effects in the model using a bootstrapping method (1000 times; Shrout \& Bolger, 2002; Bollen \& Stine, 1990). Model performance was assessed with various fit criteria, including the chi-square test, comparative fit index (CFI; Bentler,

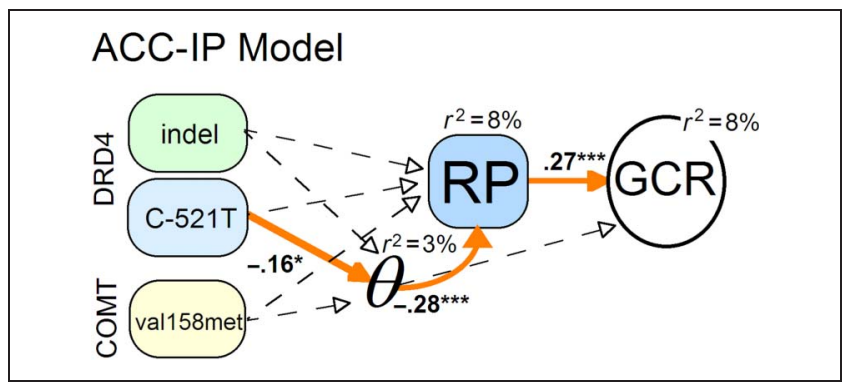

Figure 2. SEM results. Paths constituting statistically significant indirect effects are highlighted in orange. Nonsignificant paths are shown as dashed lines. Significant standardized regression coefficients for direct effects are provided along paths $(* p<.05, * * * p<.001)$, and explained variances are provided $\left(r^{2}\right)$. Full model results are provided in Table S1-S4. $\theta=$ theta power; GCR = GCR score of the World Health Organization ASSIST (Humeniuk et al., 2008). The model includes the DRD4 C-521T, DRD4 - 127 Indel SNPs, and COMT Val156Met polymorphisms.
1990), goodness-of-fit test (GFI; Tabachnick \& Fidell, 2007), and the root mean square error of approximation (RMSEA; Browne \& Cudeck, 1993). The CFI and the RMSEA are sensitive to model misspecification and are minimally affected by sample size (Hu \& Bentler, 1995). The CFI ranges from 0 to 1 , with .90 indicating acceptable fit (Bentler, 1990). RMSEA fit values less than .05 indicate close fit, and values less than .10 indicate reasonable fit (Steiger, 1990). The GFI explains what proportion of the variance/covariance patterns in the sample variance is accounted for by the model matrix; GFI values should exceed .9 for a "reasonable" model and .95 for an "impressive" model (Tabachnick \& Fidell, 2007).

In the case where model fit was poor, we performed post hoc model modifications using the modification indices (MIs) provided by the AMOS program to identify better-fitting models (Schumacker \& Lomax, 2004). An MI represents the expected decrease of the chi-square statistic if that pair of variables were allowed to covary in the model. Thus, variables exhibiting high MIs likely contribute to multicollinearity and as such are good candidates for creating a covariance path between them, which can improve the model fit without affecting the model's theoretical interpretation (Hooper, Coughlan, $\&$ Mullen, 2008). The post hoc modifications were based on empirically and theoretically sound criteria, as described below. To maintain a family-wise Type 1 error rate of $5 \%$, we modified only model variables with MIs greater than 12.83 (Tabachnick \& Fidell, 2007).

\section{RESULTS \\ Participants}

Participants (147 women, 48 men) were $20.4(S D=4.1$, range $=18-51$ ) years old on average (Table 2 ), and $91 \%$ of the sample was white. Although the sample was predominantly female, comparable to our first study (Baker et al., 2011), no gender differences were observed between RP amplitude, $t(193), 1.3, p>.1$, or theta power, $t(193), 0.99, p>1$ ), indicating that gender was not a confounding factor. Genotype groups did not differ with regard to gender, age, or ethnicity ( $p>.05)$; therefore, it is unlikely that the following analyses were confounded by population stratification. Furthermore, our sample showed no deviation from Hardy-Weinberg equilibrium (Rodriguez, Gaunt, \& Day, 2009) expectations at any of the selected loci for the overall sample $(p>.05)$, indicating that the observed genotype frequencies are consistent with previous studies of primarily white, unselected samples. Behavioral measures for the T-maze task can be found in the supplementary online material (SOM).

\section{SEM}

Figure 2 depicts the SEM results. The GFIs indicated that the hypothesized IP model provided a very strong 
Table 2. Demographic Characteristics of the Research Sample Population

\begin{tabular}{|c|c|c|c|c|c|}
\hline & \multirow[b]{2}{*}{ Median } & \multirow[b]{2}{*}{ Mean } & \multirow[b]{2}{*}{ Standard Deviation } & \multicolumn{2}{|c|}{ Range } \\
\hline & & & & Min & $\operatorname{Max}$ \\
\hline Age & 19.0 & 20.1 & 4.3 & 18 & 51 \\
\hline GCR (max: 208) & 24.0 & 28.3 & 21.0 & 1 & 104 \\
\hline Validation study ${ }^{\mathrm{a}}$ & 23.0 & 27.3 & 19.6 & 1 & 141 \\
\hline $\mathrm{RP}(\mu \mathrm{V})$ & -5.10 & -4.71 & 3.54 & -20.93 & 1.64 \\
\hline Total theta power $(\mathrm{dB})$ & 0.61 & 0.70 & 0.43 & -0.63 & 2.26 \\
\hline
\end{tabular}

${ }^{\mathrm{a}}$ Humeniuk et al. (2008), $n=1047$.

fit to the GCR scores as per conventional criteria $\left(\chi^{2}=\right.$ $4.74, d f=6, p=.577, \mathrm{CFI}=.99$, GFI $=.99$, RMSEA $<$ $.001,90 \%$ CI $[<0.001,0.07], \chi^{2} / d f$ ratio $\left.=.80\right)$, explaining approximately $8 \%$ of individual variance in GCR. No model modifications were necessary. Critically, the RP strongly predicted participants' GCR score (beta $=.27, p<.001$ ), indicating that, as RP amplitude decreased, the level of problematic substance use increased; Figure 3 illustrates the reduction in $\mathrm{RP}$ amplitude when participants were grouped categorically according to levels of drug misuse, replicating a previous finding (Baker et al., 2011; see also SOM). Note that visual inspection of the raw ERP waveforms to Reward and No-reward feedback for SD and nondependent (ND) individuals indicates that this difference is driven in the SD group by a reduced positive deflection to rewards, replicating findings from our pre- vious addiction study (Baker et al., 2011). Critically, in both studies, the negative deflections to No-reward feedback were virtually identical between SD and ND groups, confirming that the effect of interest was isolated to the predicted ERP component, the RP.

In turn, theta power contributed significantly to the amplitude of the RP (beta $=-.28, p<.001$ ), consistent with previous observations (Hajihosseini \& Holroyd, 2013). Although the genotypes together did not reliably predict RP amplitude, the model revealed that the promoter C-521T polymorphism of the DRD 4 gene uniquely predicted theta power (beta $=-.16, p<.05$ ), indicating that an increase in $\mathrm{C}$ alleles (increase in $\mathrm{D} 4$ ) predicted less theta power (Figure 4, bottom), consistent with previous findings (Marco-Pallares et al., 2009). Furthermore, the relationship between theta power and DRD4-521
Figure 3. Grand-averaged ERPs associated with Reward (blue dotted lines) and No-reward (red dashed lines) outcomes and RP (black solid lines). For the purpose of illustration, the ERPs of participants with GCR scores falling within the bottom quartile (solid lines, $n=58$; GCR score $<16$, labeled ND) and top quartile (dashed lines, $n=43$; GCR score $>41$, labeled SD) are presented (see also SOM). These scores are comparable to the cutoffs established in previous validation studies of the ASSIST for nondependence (score < 15) and dependence (score $>$ 39.5; Humeniuk et al., 2008) as well as our previous study (Baker et al., 2011). Negative voltages are plotted up by convention. (Top right) Scalp voltage maps associated with the peak value of the difference waves for ND (left) and SD (right) grand averages.

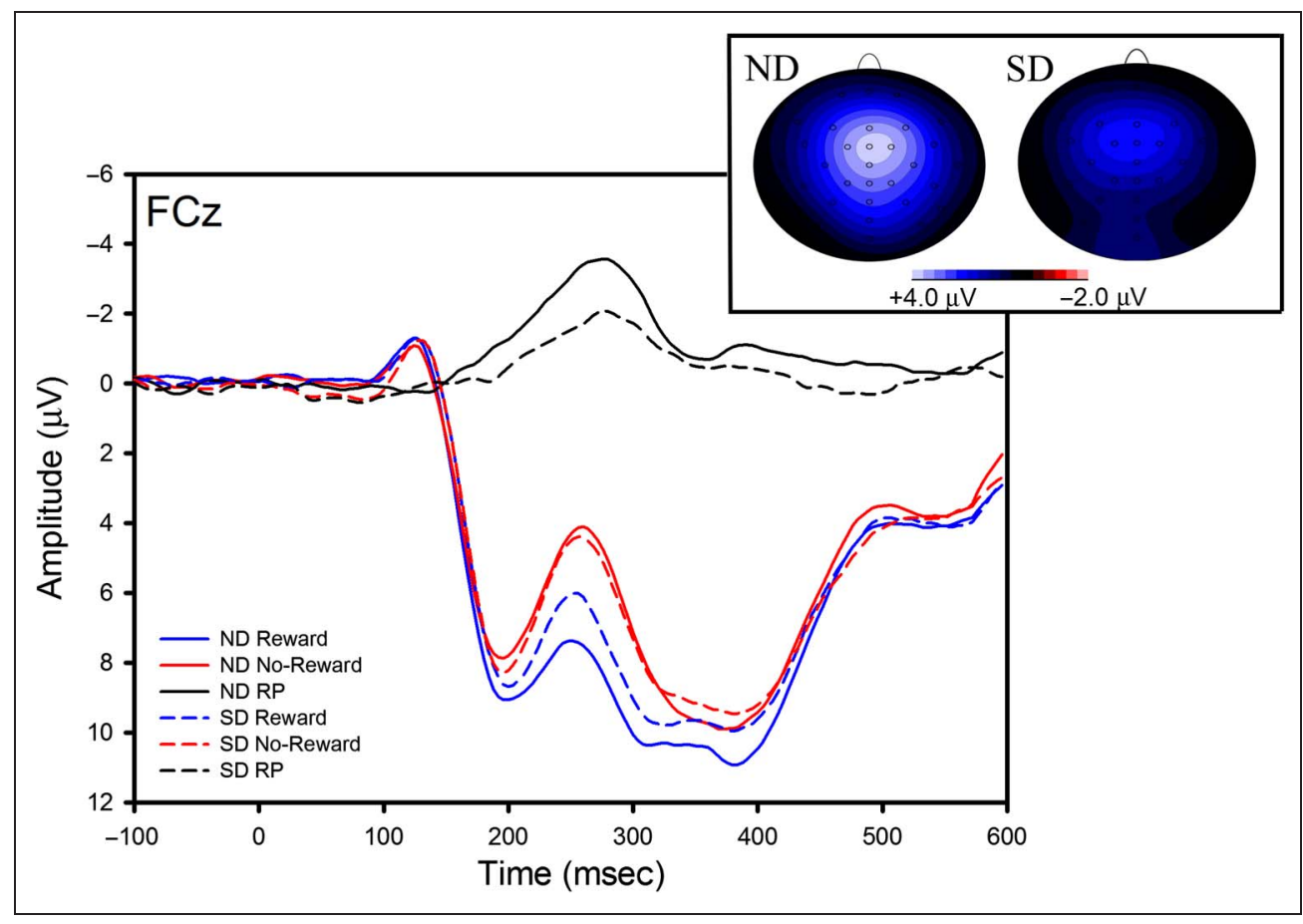


Figure 4. Time-frequency analysis of the EEG associated with outcome processing. Panel indicates changes in power for each frequency band by condition (top left: Reward, top right: No-reward, bottom left: total power) with respect to baseline. (Bottom right) DRD4 C-521T gene dose effect on theta power. Individuals with more $\mathrm{T}$ alleles displayed relatively more theta power after feedback onset. EEG data recorded at channel FCz.

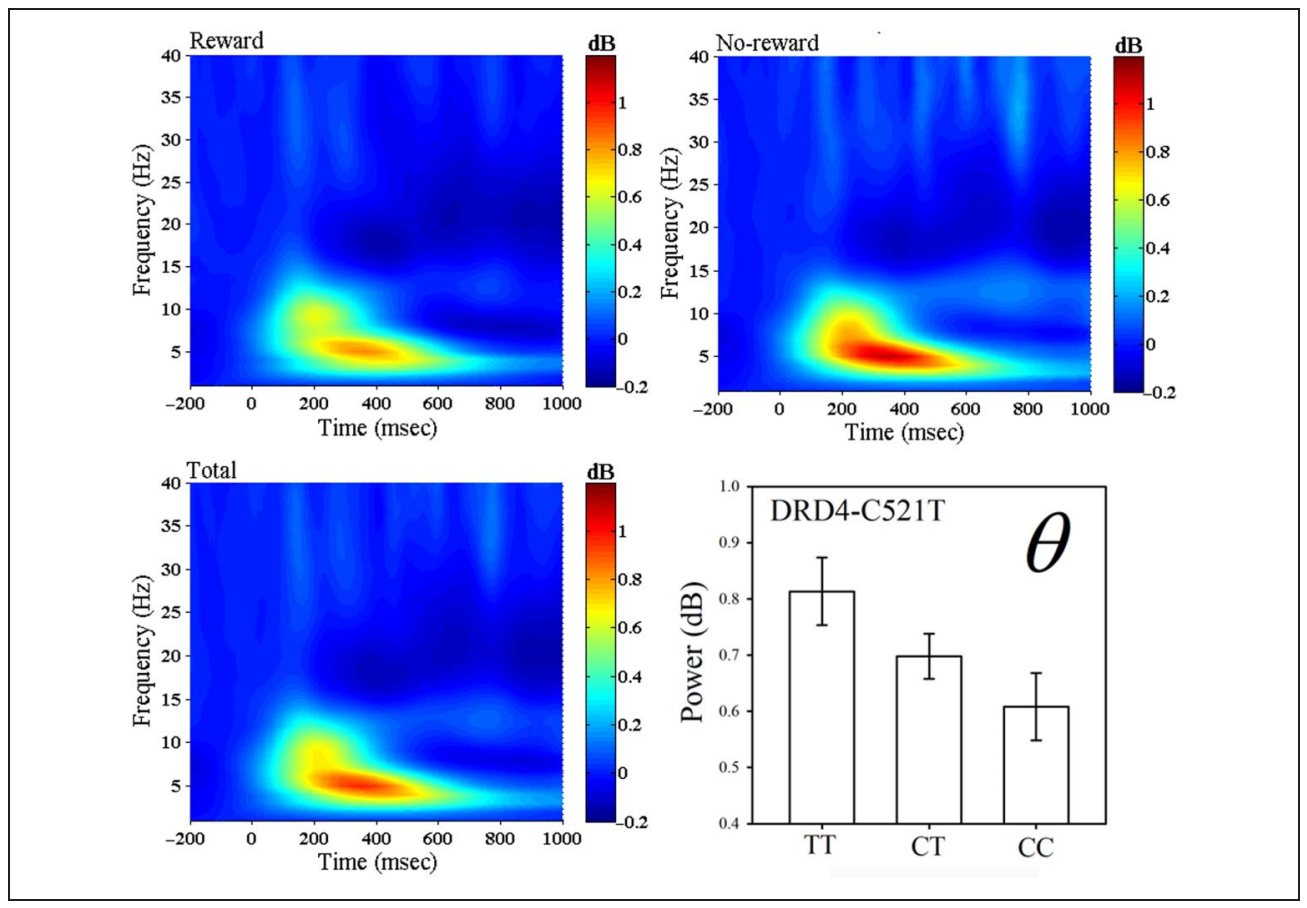

expression was strikingly similar across Reward and Noreward conditions (theta Reward, $F(2,192)=2.89, p<$ .05 ; theta No-reward, $F(2,192)=2.89, p<.05)$, indicating that individuals carrying the $\mathrm{T}$ allele expressed more theta power than did individuals carrying the $\mathrm{C}$ allele, irrespective of the condition. Thus, the D4 receptor appears to play a general, nonspecific inhibitory role in pFC. Finally, the indirect effect from the DRD4 C-521T genotype to the RP through theta was statistically significant $(p=.012)$, and the indirect effect from theta power to GCR score through the RP was statistically significant $(p=.002)$, indicating that the variation in DRD4-521 gene can influence the level of substance dependence indirectly through the degree of engagement of ACC. ${ }^{3}$

\section{DISCUSSION}

We applied SEM to investigate whether two electrophysiological phenomena related to the cognitive control function of ACC (RP amplitude and theta power) can serve as an IP for substance misuse. Our model results point to a specific molecular pathway by which the DRD4-521 genotype alters ACC electrophysiology and thereby predicts the level of problematic substance use in undergraduate students. To be specific, the RP (Warren, Hyman, Seamans, \& Holroyd, 2015; Proudfit, 2015; Emeric et al., 2008) and frontal midline theta oscillations (Cavanagh \& Frank, 2014) are believed to be produced within caudal ACC. A detailed examination of these phenomena has indicated that theta power reflects a "default" response by ACC to unexpected task-relevant events (Holroyd, Larsen, \& Cohen, 2004) and that the RP is produced by dopamine-dependent RPE signals to unexpected rewarding outcomes that suppress the default response (Hajihosseini \& Holroyd, 2013). Furthermore, a recent theory holds that ACC uses dopamine reward signals for learning the value of extended, context-specific sequences of behavior directed toward particular goals (Holroyd \& Yeung, 2012). Evaluated in this context, our findings of reduced RP amplitude in individuals who tend to misuse drugs of abuse suggest abnormal goal-directed behavior in this population (Baker, 2012; Baker et al., 2011).

Furthermore, the fact that these electrophysiological phenotypes were selectively modulated by a DRD4-related genetic polymorphism specifically implicates ACC D4 receptors in addiction. D4 receptors are highly expressed in frontal regions for cognitive control including ACC and pFC (Mulcrone \& Kerwin, 1997), where their activation by tonic dopamine inhibits neural firing (Onn et al., 2006; Rubinstein et al., 2001). These receptors appear partly responsible for regulating behavioral shifts between different task strategies (Floresco \& Magyar, 2006; see also Holroyd \& McClure, 2015). For example, high levels of D4 activity in rodents can impair performance on goalorientated set-shifting tasks (by increasing the commission of perseverative errors), whereas less D4 activity can lead to an improvement, suggesting that D4 receptor activation may antagonize phasic activity underlying behavioral flexibility (Floresco \& Magyar, 2006). Consistent with this evidence, human electrophysiological studies have shown that the $\mathrm{T}$ allele of the promoter -521 (C/T) SNP (rs1800955) of the DRD4 gene, which is associated with $40 \%$ less transcriptional efficiency of D4 receptors (Okuyama, Ishiguro, Toru, \& Arinami, 1999; but see Kereszturi et al., 
2006), impacts frontal cortex functioning related to cognitive control (Kramer et al., 2007).

In this study, we found that $\mathrm{C}$ allele carriers associated with enhanced D4 expression (Okuyama et al., 1999; but see Kereszturi et al., 2006) of the DRD4 promoter -521 SNP displayed a relatively suppressed theta response by medial frontal cortex to salient or unexpected taskrelevant events. In turn, there was an indirect effect of theta on GCR score through the RP. In line with the hierarchical reinforcement learning theory of ACC (Holroyd \& McClure, 2015; Holroyd \& Yeung, 2012), this indirect pathway suggests that $\mathrm{D} 4$ receptors may play a pivotal role in decision-making over extended behaviors, a process that goes awry in substance use disorders. We speculate that, whereas too many $\mathrm{D} 4$ receptors would suppress the normal reinforcement learning function of ACC, the dopamine-potentiating effects of addictive substances would compound this problem, resulting in unstable reward valuation as revealed by our electrophysiological measures. Conversely, decreased D4 expression could promote optimal ACC function by enabling it to respond dynamically to reinforcement signals such as by switching to a new behavioral state. This function is suggested by the relatively strong theta response of medial frontal cortex to salient events, which evidently reflects the release of ACC from tonic dopamine-induced inhibition. According to this account, a genotype associated with stronger cognitive control may act as a protective mechanism against the dopamine-potentiating effects of addictive substances.

This study raises several issues related to the relationship between dopamine-related SNPs and electrophysiological effects related to cognitive control. First, whereas previous studies have reported links between ERPs related to performance monitoring (response-locked errorrelated negativity, feedback error-related negativity) and dopamine-related genes (i.e., DRD4, COMT; for a review, see Manoach \& Agam, 2013; see also Agam et al., 2014), in this study, none of the genotypes significantly predicted $\mathrm{RP}$ amplitude. Most candidate gene investigations have analyzed condition-specific ERPs related to error-locked ERP averages (e.g., error-related negativity, feedback-error related negativity), whereas here we quantified the size of the RP using a subtraction method, which could have contributed to this inconsistency across studies. Furthermore, because the data averaging process underlying the ERP approach can obscure oscillatory signals that vary in phase across trials (Tallon-Baudry \& Bertrand, 1999), our results suggest that time-frequency analysis (which is less sensitive to such latency variability) might better reveal D4-dependent electrophysiological effects related to cognitive control. Second, previous studies have focused on candidate genes (DRD4, COMT) that are implicated in general processes related to cognitive control (e.g., error processing; Marco-Pallares et al., 2009; Kramer et al., 2007) but not necessarily related to reward processing per se. Our theoretical framework suggests that the RP may be more closely linked to genes that are associated with the proposed reward function of ACC (e.g., the DRD1 SNP [rs686]; e.g., Baker et al., 2015). Relatedly, the relationship between DRD-521 SNP and D4 expression is still debated, in part because the gene is highly polymorphic (Kereszturi et al., 2006). Future studies could investigate other polymorphisms of the DRD4 promoter region in the context of different genetic haplotypes, such as the SNPs in positions $-768,-616,-615,-603$, and -600 , which may also influence promoter activity (Kereszturi et al., 2006).

A limitation of the study is that the sample consisted of undergraduate students recruited from the University of Victoria who may not be representative of the population in general nor of severely dependent substance users in particular. However, nearly a quarter of the sample met the ASSIST criteria for substance dependence. Furthermore, the mean GCR score for this SD group (61) approached the mean GCR score of individuals who completed several weeks of treatment for substance dependence (83; Baker et al., 2013), indicating that this subgroup expressed elevated levels of problematic use of addictive substances. For this reason, we believe that this undergraduate population provides a reasonable point of departure for examining substance misuse in other populations. Note also that the GCR score of the WHO ASSIST identifies individuals who tend to abuse multiple substances rather than individuals who present a pure clinical case. Given the high prevalence of polysubstance abuse in young adults between the ages of 18 and 24 years (Barrett, Darredeau, \& Pihl, 2006) and that individuals who abuse only a single addictive drug comprise only a small segment of the general population, we believe that this operational definition of substance misuse is appropriate. Our study thus reveals that individuals who abuse multiple substances share important neural abnormalities in reward processing and cognitive control irrespective of their particular drugs of choice. These results can be investigated further in future studies that expand the sample to include other populations of users who abuse particular drugs or drug types (such as stimulants vs. sedatives).

In summary, our results suggest that genetically determined overexpression of D4 receptors in medial frontal cortex disrupts the association of extended, goal-directed behaviors with positive value (Holroyd \& McClure, 2015; Holroyd \& Yeung, 2012), conferring vulnerability to the potentiating effects of addictive drugs. These findings motivate further study of the role of cognitive control in mediating the impact of dopamine-related genetic polymorphisms on psychopathology and suggest how future interventions could be individually tailored for specific genetic and neurocognitive profiles. For instance, we speculate that excessive dopaminergic inhibition of frontal cortex, particularly in individuals who carry the $\mathrm{C}$ allele of the DRD4-521 SNP, might be alleviated by administration of pharmaceuticals (such as D4 antagonists) 
or neuromodulation techniques (such as TMS; e.g., Baker et al., 2015), in addition to application of cognitivebehavioral therapy to develop better coping strategies. By identifying how the brain links genes to addiction, novel treatments and early diagnosis for substance use disorders may finally be on the horizon.

\section{Acknowledgments}

This research was supported by Canadian Institutes of Health Research Operating Grant 97750 awarded to C. B. H. The first author was supported by Doctoral Awards from the Integrated Mentor Program in Addictions Research Training and the Canadian Institutes of Health Research no. 195501. We are grateful to Patrick Macleod, Mike Hunter, and Stuart Macdonald for their consultation on this project as well as to Somayyeh Montazer-Hojat, Elizabeth Plant, and the research assistants of the Learning and Cognitive Control Laboratory for help with data collection.

Reprint requests should be sent to Travis E. Baker, Centre de Recherche du CHU Ste-Justine, Université de Montréal, 3175 Chemin de la Côte Sainte-Catherine, Montreal, H3T 1C5, Canada, or via e-mail: travis.e.baker@gmail.ca.

\section{Notes}

1. Rasetti and Weinberger (2011) state, "To link a gene effect in brain to the gene effect on risk for the syndromal diagnosis, it is necessary to show that the brain effect is a biological substrate also linked to risk, a so-called intermediate phenotype" (p. 340).

2. Cognitive control, broadly defined, refers to a collection of cognitive processes related to the regulation of thought, emotion, and actions in the service of achieving current and future goals (i.e., goal-directed behavior; Miller \& Cohen, 2001).

3. Because of recent controversy about the construct validity of the RP with respect to frontal midline theta oscillations (Hajihosseini \& Holroyd, 2013; Holroyd, Hajihosseini, \& Baker, 2012; Cohen, Wilmes, \& van de Vijver, 2011), we also analyzed competing models and compared the results against our proposed model. These alternative models included a model that excluded the RP IP, a model that included theta power separately for Reward and No-reward feedback (while excluding the RP), and a model that included paths between genes and GCR. Overall, model comparisons indicated that our hypothesized ACC-IP model provided the best fit to the data when compared with alternative models (see SOM).

\section{REFERENCES}

Agam, Y., Vangel, M., Roffman, J. L., Gallagher, P. J., Chaponis, J., Haddad, S., et al. (2014). Dissociable genetic contributions to error processing: A multimodal neuroimaging study. PLOS ONE, 9, e101784.

Arbuckle, J. L. (1995-2009). Amos 18 user's guide. Chicago, IL: SPSS.

Baker, T. E. (2012). Genetics, drugs, and cognitive control: Uncovering individual differences in substance dependence. $\mathrm{PhD}$ dissertation, Brain and Cognitive Science, University of Victoria.

Baker, T. E., \& Holroyd, C. B. (2009). Which way do I go? Neural activation in response to feedback and spatial processing in a virtual T-maze. Cerebral Cortex, 19, 1708-1722.

Baker, T. E., Stockwell, T., Barnes, G., \& Holroyd, C. B. (2011). Individual differences in substance dependence: At the intersection of brain, behaviour and cognition. Addiction Biology, 16, 458-466.

Baker, T. E., Stockwell, T., \& Holroyd, C. B. (2013). Constraints on decision making: Implications from genetics, personality, and addiction. Cognitive, Affective \& Behavioral Neuroscience, 13, 417-436.

Baker, T. E., Tucholka, A., Potvin, S., Lesperance, P., JutrasAswad, D., Larcher, K., et al. (2015). Optimizing combined fMRI-DTI-TMS-ERP methods to identify and regulate reward valuation during nicotine craving. Poster presented at the 21st Annual Meeting of the Organization for Human Brain Mapping, Honolulu, HI.

Baransel Isir, A. B., Oguzkan, S., Nacak, M., Gorucu, S., Dulger, H. E., \& Arslan, A. (2008). The catechol-O-methyl transferase Val158Met polymorphism and susceptibility to cannabis dependence. American Journal of Forensic Medicine and Pathology, 29, 320-322.

Barrett, S. P., Darredeau, C., \& Pihl, R. O. (2006). Patterns of simultaneous polysubstance use in drug using university students. Human Psychopharmacology: Clinical and Experimental, 21, 255-263.

Bentler, P. M. (1990). Comparative fit indices in structural models. Psychological Bulletin, 107, 238-246.

Beuten, J., Payne, T. J., Ma, J. Z., \& Li, M. D. (2006). Significant association of catechol-O-methyltransferase (COMT) haplotypes with nicotine dependence in male and female smokers of two ethnic populations.

Neuropsychopharmacology, 31, 675-684.

Bollen, K. A., \& Stine, R. A. (1990). Direct and indirect effects: Classical and bootstrap estimates of variability. Sociological Methodology, 20, 115-140.

Browne, M. W., \& Cudeck, R. (1993). Alternative ways of assessing model fit. In K. Bollen \& K. Long (Eds.) pp. 136-162.

Cavanagh, J. F., \& Frank, M. J. (2014). Frontal theta as a mechanism for cognitive control. Trends in Cognitive Sciences, 18, 414-421.

Cavanagh, J. F., \& Shackman, A. J. (2015). Frontal midline theta reflects anxiety and cognitive control: Meta-analytic evidence. Journal of Physiology, Paris, 109, 3-15.

Cavanagh, J. F., Zambrano-Vazquez, L., \& Allen, J. J. (2012). Theta lingua franca: A common mid-frontal substrate for action monitoring processes. Psychophysiology, 49, 220-238.

Chen, J., Lipska, B. K., Halim, N., Ma, Q. D., Matsumoto, M. Melhem, S., et al. (2004). Functional analysis of genetic variation in catechol-O-methyltransferase (COMT): Effects on mRNA, protein, and enzyme activity in postmortem human brain. American Journal of Human Genetics, 75, 807-821.

Cohen, M. X., Wilmes, K. \& van de Vijverl, I. (2011). Cortical electrophysiological network dynamics of feedback learning. Trends in Cognitive Sciences, 15, 558-566.

Cuthbert, B. N., \& Insel, T. R. (2013). Toward the future of psychiatric diagnosis: The seven pillars of RDoC. $B M C$ Medicine, 11, 126.

Di Chiara, G., \& Bassareo, V. (2007). Reward system and addiction: What dopamine does and doesn't do. Current Opinion in Pharmacology, 7, 69-76.

Di Chiara, G., \& Imperato, A. (1988). Drugs abused by humans preferentially increase synaptic dopamine concentrations in the mesolimbic system of freely moving rats. Proceedings of the National Academy of Sciences, U.S.A., 85, 5274-5278.

Dickinson, D., \& Elvevag, B. (2009). Genes, cognition and brain through a COMT lens. Neuroscience, 164, 72-87.

Emeric, E. E., Brown, J. W., Leslie, M., Pouget, P., Stuphorn, V., \& Schall, J. D. (2008). Performance monitoring local field potentials in the medial frontal cortex of primates: Anterior cingulate cortex. Journal of Neurophysiology, 99, 759-772. 
Fan, J., Fossella, J., Sommer, T., Wu, Y., \& Posner, M. I. (2003). Mapping the genetic variation of executive attention onto brain activity. Proceedings of the National Academy of Sciences, U.S.A., 100, 7406-7411.

Floresco, S. B., \& Magyar, O. (2006). Mesocortical dopamine modulation of executive functions: Beyond working memory. Psychopharmacology, 188, 567-585.

Frank, M. J., Seeberger, L. C., \& O'Reilly, R. C. (2004). By carrot or by stick: Cognitive reinforcement learning in parkinsonism. Science, 306, 1940-1943.

Gaspar, P., Berger, B., Febvret, A., Vigny, A., \& Henry, J. P. (1989). Catecholamine innervation of the human cerebral cortex as revealed by comparative immunohistochemistry of tyrosine hydroxylase and dopamine-beta-hydroxylase. Journal of Comparative Neurology, 279, 249-271.

Gratton, G., Coles, M. G., \& Donchin, E. (1983). A new method for off-line removal of ocular artifact. Electroencephalography Clinical Neurophysiology, 55, 468-484.

Hajihosseini, A., \& Holroyd, C. B. (2013). Frontal midline theta and N200 amplitude reflect complementary information about expectancy and outcome evaluation. Psychophysiology, 50, 550-562.

Holroyd, C. B. (2015). The waste disposal problem of effortful control. In T. Braver (Ed.), Motivation and cognitive control (pp. 235-260). New York: Psychology Press.

Holroyd, C. B., \& Coles, M. G. (2002). The neural basis of human error processing: Reinforcement learning, dopamine, and the error-related negativity. Psychological Review, 109, 679-709.

Holroyd, C. B., HajiHosseini, A., \& Baker, T. E. (2012). ERPs and EEG oscillations, best friends forever: Comment on Cohen et al. Trends in Cognitive Sciences, 16, 192.

Holroyd, C. B., \& Krigolson, O. E. (2007). Reward prediction error signals associated with a modified time estimation task. Psychophysiology, 44, 913-917.

Holroyd, C. B., Krigolson, O. E., \& Lee, S. (2011). Reward positivity elicited by predictive cues. NeuroReport, 22, 249-252

Holroyd, C. B., Larsen, J. T. \& Cohen, J. D. (2004). Context dependence of the eventrelated brain potential associated with reward and punishment. Psychophysiology, 41, 245-253.

Holroyd, C. B., \& McClure, S. M. (2015). Hierarchical control over effortful behavior by rodent medial frontal cortex: A computational model. Psychological Review, 122, 54-83.

Holroyd, C. B., Pakzad-Vaezi, K. L., \& Krigolson, O. E. (2008). The feedback correct-related positivity: Sensitivity of the event-related brain potential to unexpected positive feedback. Psychophysiology, 45, 688-697.

Holroyd, C. B., \& Umemoto, A. (2015). Anterior cingulate function and dysfunction in health and disease. Conference Abstract-Psychophysiology, 52, S37.

Holroyd, C. B., \& Yeung, N. (2012). Motivation of extended behaviors by anterior cingulate cortex. Trends in Cognitive Sciences, 16, 122-128.

Hooper, D., Coughlan, J. \& Mullen, M. R. (2008). Structural equation modelling: Guidelines for determining model fit. Journal of Business Research Methods, 6, 53-60.

Hsieh, L. T., \& Ranganath, C. (2014). Frontal midline theta oscillations during working memory maintenance and episodic encoding and retrieval. Neuroimage, 85, 721-729.

Humeniuk, R., Ali, R., Babor, T. F., Farrell, M., Formigoni, M. L., Jittiwutikarn, J., et al. (2008). Validation of the Alcohol, Smoking and Substance Involvement Screening Test (ASSIST). Addiction, 103, 1039-1047.

Hu, L., \& Bentler, P. (1995). Evaluating model fit. In R. H. Hoyle (Ed.), Structural equation modeling: Concepts, issues, and applications (pp. 76-99). Thousand Oaks, CA: Sage.
Hutchison, K. E., McGeary, J., Smolen, A., Bryan, A., \& Swift, R. M. (2002). The DRD4 VNTR polymorphism moderates craving after alcohol consumption. Health Psychology, 21, 139-146.

Hyman, S. E., Malenka, R. C., \& Nestler, E. J. (2006). Neural mechanisms of addiction: The role of reward-related learning and memory. Annual Review of Neuroscience, 29, 565-598.

Insel, T., Cuthbert, B., Garvey, M., Heinssen, R., Pine, D. S., Quinn, K., et al. (2010). Research domain criteria (RDoC): Toward a new classification framework for research on mental disorders. American Journal of Psychiatry, 167, 748-751.

Jasper, H. H. (1958). The ten twenty electrode system of the international federation. Electroencephalography and Clinical Neurophysiology, 10, 371.

Johnson, C., Drgon, T., Liu, Q. R., Zhang, P. W., Walther, D., Li, C. Y., et al. (2008). Genome wide association for substance dependence: Convergent results from epidemiologic and research volunteer samples. BMC Medical Genetics, 9, 113.

Kereszturi, E., Kiraly, O., Barta, C., Molnar, N., Sasvari-Szekely, M., \& Csapo, Z. (2006). No direct effect of the $-521 \mathrm{C} / \mathrm{T}$ polymorphism in the human dopamine $\mathrm{D} 4$ receptor gene promoter on transcriptional activity. BMC Molecular Biology, 7, 18

Kramer, U. M., Cunillera, T., Camara, E., Marco-Pallares, J., Cucurell, D., Nager, W., et al. (2007). The impact of catechol$\mathrm{O}$-methyltransferase and dopamine $\mathrm{D} 4$ receptor genotypes on neurophysiological markers of performance monitoring. Journal of Neuroscience, 27, 14190-14198.

Lachaux, J. P., Rodriguez, E., Martinerie, J., \& Varela, F. J. (1999). Measuring phase synchrony in brain signals. Human Brain Mapping, 8, 94-208.

Lenzenweger, M. F. (2013). Endophenotype, intermediate phenotype, biomarker: Definitions, concept comparisons, clarifications. Depression and Anxiety, 30, 185-189.

Lima, D. R., Goncalves, P. D., Malbergier, A., Amaral, R., Andrade, A. G., \& Cunha, P. J. (2015). The DSM-5 and the diagnosis of substance use disorders: Reflection about validity of the new criteria and possible 'missing pieces' in the puzzle. Australian and New Zealand Journal of Psychiatry, 49, 940-941.

Loth, E., Carvalho, F., \& Schumann, G. (2011). The contribution of imaging genetics to the development of predictive markers for addictions. Trends in Cognitive Sciences, 15, 436-446.

Mackillop, J., Menges, D. P., McGeary, J. E., \& Lisman, S. A. (2007). Effects of craving and DRD4 VNTR genotype on the relative value of alcohol: An initial human laboratory study. Behavioral and Brain Functions, 3, 11.

Manoach, D. S., \& Agam, Y. (2013). Neural markers of errors as endophenotypes in neuropsychiatric disorders. Frontiers in Human Neuroscience, 7, 350.

Marco-Pallares, J., Cucurell, D., Cunillera, T., Kramer, U. M., Camara, E., Nager, W., et al. (2009). Genetic variability in the dopamine system (dopamine receptor D4, catechol-O-methyltransferase) modulates neurophysiological responses to gains and losses. Biological Psychiatry, 66, 154-161.

Marco-Pallarés, J., Nager, W., Krämer, U. M., Cunillera, T., Càmara, E., \& Cucurell, D. (2010). Neurophysiological markers of novelty processing are modulated by COMT and DRD4 genotypes. Neuroimage, 53, 962-969.

McGeary, J. (2009). The DRD4 exon 3 VNTR polymorphism and addiction-related phenotypes: A review. Pharmacology, Biochemistry and Behavior, 93, 222-229.

Meyer-Lindenberg, A., \& Weinberger, D. R. (2006). Intermediate phenotypes and genetic mechanisms of psychiatric disorders. Nature Reviews Neuroscience, 7, 818-827. 
Miller, E. K., \& Cohen, J. D. (2001). An integrative theory of prefrontal cortex function. Annual Review of Neuroscience, 24, 167-202.

Miltner, W. H. R., Braun, C. H., \& Coles, M. G. H. (1997). Eventrelated brain potentials following incorrect feedback in a time-estimation task: Evidence for a 'generic' neural system for error detection. Journal of Cognitive Neuroscience, 9, 788-798.

Mitchell, D. J., McNaughton, N., Flanagan, D., \& Kirk, I. J. (2008) Frontal-midline theta from the perspective of hippocampal "theta". Progress in Neurobiology, 86, 156-185.

Mulcrone, J., \& Kerwin, R. W. (1997). The regional pattern of D4 gene expression in human brain. Neuroscience Letters, 234, 147-150.

Newcombe, D. A., Humeniuk, R. E., \& Ali, R. (2005). Validation of the World Health Organization Alcohol, Smoking and Substance Involvement Screening Test (ASSIST): Report of results from the Australian site. Drug Alcohol Review, 24, 217-217.

Oak, J. N., Oldenhof, J., \& Van Tol, H. H. (2000). The dopamine D(4) receptor: One decade of research. European Journal of Pharmacology, 405, 303-327.

Okuyama, Y., Ishiguro, H., Toru, M., \& Arinami, T. (1999). A genetic polymorphism in the promoter region of DRD4 associated with expression and schizophrenia. Biochemical and Biophysical Research Communications, 258, 292-295.

Olvet, D. M., \& Hajcak, G. (2008). The error-related negativity (ERN) and psychopathology: Toward an endophenotype. Clinical Psychology Review, 28, 1343-1354.

Onn, S. P., Wang, X. B., Lin, M., \& Grace, A. A. (2006). Dopamine D1 and D4 receptor subtypes differentially modulate recurrent excitatory synapses in prefrontal cortical pyramidal neurons. Neuropsychopharmacology, 31, 318-338.

Oosterhuis, B. E., LaForge, K. S., Proudnikov, D., Ho, A., Nielsen, D. A., Gianotti, R., et al. (2008). Catechol-O-methyltransferase (COMT) gene variants: Possible association of the Val158Met variant with opiate addiction in Hispanic women. American Journal of Medical Genetics, Part B: Neuropsychiatric Genetics, 147B, 793-798.

Peoples, L. L. (2002). Neuroscience. Will, anterior cingulate cortex, and addiction. Science, 296, 1623-1624.

Proudfit, G. H. (2015). The reward positivity: From basic research on reward to a biomarker for depression. Psychophysiology, 52, 449-459.

Rasetti, R., \& Weinberger, D. R. (2011). Intermediate phenotypes in psychiatric disorders. Current Opinion in Genetics and Development, 21, 340-348.

Ray, L. A., Bryan, A., Mackillop, J., McGeary, J., Hesterberg, K., \& Hutchison, K. E. (2009). The dopamine D receptor (DRD4) gene exon III polymorphism, problematic alcohol use and novelty seeking: Direct and mediated genetic effects. Addiction Biology, 14, 238-244.

Redish, A. D. (2004). Addiction as a computational process gone awry. Science, 306, 1944-1947.

Redish, A. D., Jensen, S., \& Johnson, A. (2008). A unified framework for addiction: Vulnerabilities in the decision process. Behavioral and Brain Sciences, 31, 415-437.

Rice, M. E., \& Cragg, S. J. (2004). Nicotine amplifies rewardrelated dopamine signals in striatum. Nature Neuroscience, 7, 583-584.

Rodriguez, S., Gaunt, T. R., \& Day, I. N. (2009). Hardy-Weinberg equilibrium testing of biological ascertainment for Mendelian randomization studies. American Journal of Epidemiology, 169, 505-514.

Rubinstein, M., Cepeda, C., Hurst, R. S., Flores-Hernandez, J., Ariano, M. A., Falzone, T. L., et al. (2001). Dopamine D4 receptor-deficient mice display cortical hyperexcitability. Journal of Neuroscience, 21, 3756-3763.
Sambrook, T. D., \& Goslin, J. (2015). A neural reward prediction error revealed by a meta-analysis of ERPs using great grand averages. Psychological Bulletin, 141, $213-235$.

Schultz, W. (2010). Dopamine signals for reward value and risk: Basic and recent data. Behavioral and Brain Functions, $6,24$.

Schultz, W. (2011). Potential vulnerabilities of neuronal reward, risk, and decision mechanisms to addictive drugs. Neuron, 69, 603-617.

Schultz, W. (2013). Updating dopamine reward signals. Current Opinion in Neurobiology, 23, 229-238.

Schumacker, R. E. \& Lomax, R. G. (2004). A beginner's guide to structural equation modeling (2nd ed.). Mahwah, NJ: Lawrence Erlbaum.

Shrout, P. E., \& Bolger, N. (2002). Mediation in experimental and nonexperimental studies: New procedures and recommendations. Psychological Methods, 7, 422-445.

Steiger, J. H. (1990). Structural model evaluation and modification: An interval estimation approach. Multivariate Behavioral Research, 25, 173-180.

Sutton, R. S., \& Barto, A. G. (1998). Reinforcement learning An introduction. IEEE Transactions on Neural Networks, 9, 1054 .

Tabachnick, B. G., \& Fidell, L. S. (2007). Using Multivariate Statistics, 5th ed. New York: Allyn and Bacon.

Tallon-Baudry, C. \& Bertrand, O. (1999). Oscillatory gamma activity in humans and its role in object representation. Trends in Cognitive Sciences, 3, 151-162.

Uhl, G. R., Drgon, T., Johnson, C., Li, C. Y., Contoreggi, C., Hess, J., et al. (2008). Molecular genetics of addiction and related heritable phenotypes: Genome-wide association approaches identify "connectivity constellation" and drug target genes with pleiotropic effects. Annals of the New York Academy of Sciences, 1141, 318-381.

Volkow, N. D., Wang, G. J., Fowler, J. S., \& Tomasi, D. (2012). Addiction circuitry in the human brain. Annual Review of Pharmacology and Toxicology, 52, 321-336.

Volkow, N. D., Wang, G. J., Fowler, J. S., Tomasi, D., \& Telang, F. (2011). Addiction: Beyond dopamine reward circuitry. Proceedings of the National Academy of Sciences, U.S.A., 108, 15037-15042.

Walsh, M. M., \& Anderson, J. R. (2012). Learning from experience: Event-related potential correlates of reward processing, neural adaptation, and behavioral choice. Neuroscience \& Biobehavioral Reviews, 36, 1870-1884.

Warren, C. M., \& Holroyd, C. B. (2012). The impact of deliberative strategy dissociates ERP components related to conflict processing vs. reinforcement learning. Frontiers in Neuroscience, 6, 43.

Warren, C. M., Hyman, J. M., Seamans, J. K., \& Holroyd, C. B. (2015). Feedback-related negativity observed in rodent anterior cingulate cortex. Journal of Physiology, Paris, 109, 87-94.

Weinberg, A., \& Hajcak, G. (2011). Longer term test-retest reliability of error-related brain activity. Psychophysiology, 48, $1420-1425$.

Yeung, N., Bogacz, R., Holroyd, C. B., \& Cohen, J. D. (2004). Detection of synchronized oscillations in the electroencephalogram: An evaluation of methods. Psychophysiology, 41, 822-832.

Yin, H. H., \& Knowlton, B. J. (2006). The role of the basal ganglia in habit formation. Nature Reviews Neuroscience, 7 , 464-476.

Zaghloul, K. A., Blanco, J. A., Weidemann, C. T., McGill, K., Jaggi, J. L., Baltuch, G. H., et al. (2009). Human substantia nigra neurons encode unexpected financial rewards. Science, 323, 1496-1499. 